\title{
Microvortices and recirculating flow generated by an oscillatory microplate for microfluidic applications
}

\author{
Cheng Ming Lin, Yu Shang Lai, Hsin Ping Liu, and Andrew M. Wo ${ }^{a)}$ \\ Institute of Applied Mechanics, National Taiwan University, Taipei 106, Taiwan
}

(Received 17 July 2008; accepted 9 September 2008; published online 29 September 2008)

\begin{abstract}
Circulatory flow structures can be useful in a microfluidic device but often are difficult to generate mechanically in microscale. This paper presents generation of such flow via an in-plane resonating microplate $\left(100 \times 100 \times 1.2 \mu \mathrm{m}^{3}\right)$ actuated by Lorentz law. Results show either one of two nonlinear time-mean flow structures is feasible for the finite plate: (1) two-dimensional (2D) small-scale, counter-rotating microvortices or (2) three-dimensional, large-scale, recirculating flow. Sharpness of microplate's edge is found to be the decisive factor for 2D microvortices to form. Both flow structures are robust and controllable. Potential applications include trapping and mixing of bioparticles in microfluidic devices. (C) 2008 American Institute of Physics.
\end{abstract}

[DOI: $10.1063 / 1.2991444]$

With much progress in microfluidics in the last decade, the technology is becoming a viable "laboratory-on-a-chip" platform for applications in biochemical assays. ${ }^{1,2}$ Advantages of the fluid handling technology include micro/nanosample volume, rapid response time, amenable to microfabrication, and ease of integration to form a total-analysis system. The convenience of this analysis platform largely depends on the ability to control fluid streams and its interaction with biosamples within the device. Application of the technology has been demonstrated in cell biology and bacterial, protein, and DNA analyses. ${ }^{3}$

Circulating flow structure has been exploited in various microfluidic applications. For example, vortical flows structure enhances mixing - difficult in microscale due to Reynolds number $\leqslant 1$-by increasing the interfacial area between heterogeneous fluid streams or encourages chaotic three-dimensional (3D) flow. Most passive micromiters generate circulating flow by passing fluid through tailored microchannel geometries. ${ }^{4,5}$ Electro-osmosis, for example, leverages on microelectrodes to induce ionic motion in the double layer and form vortices to mix, pump, and/or separation bioparticles. ${ }^{6-8}$ Other applications of circulating flow include magnetically driven microstirrer, ${ }^{9}$ separation of chiral molecules via spatially variable vorticity in a microfluidic flow, ${ }^{10}$ and dynamic assembly of electrolyte deposition. ${ }^{11}$

Steady streaming - time mean flow resulted from periodic condition-has a large body of knowledge in fluid mechanics. ${ }^{12-15}$ This technique is attractive in microdevices since periodic motion is relatively easy to generate on chip but not the steady flow. The flow phenomenon has been used to pump fluid ${ }^{16}$ and to lyse cells, ${ }^{17}$ but their usage thus far is limited mainly due to difficulty in its generation. With wide usage of circulating flow in microfluidics, further breakthrough is required to encourage the use of streaming flow toward this end.

This work presents a mechanical microdevice that generates steady streams in a short distance and essentially independent on properties of embedded bioparticles and me-

${ }^{a)}$ Electronic mail: andrew@iam.ntu.edu.tw. dium. Flow structure and relevant physics are characterized experimentally and computationally.

Design of the microdevice is based on secondary steady streaming flow structure resulted from in-plane resonant oscillation of a suspended microplate. We focus on the outer circulating flow, not the inner eddies near the solid boundary, since the penetration depth $\delta_{\mathrm{ac}} \sim(v / \omega)^{1 / 2}$ ( $v$ is the kinematic viscosity and $\omega$ the oscillation frequency) is only $\sim 6 \mu \mathrm{m}$ at $140 \mathrm{kHz}$ in water. The geometric design is a microthin plate ( $L \gg t$, where $L$ is the length of the plate and $t$ the plate thickness) oscillating in liquid with an infinitesimal displacement $(s<1 \mu \mathrm{m})$. The main structure of the microdevice consists of a suspended square microplate $(100 \times 100$ $\left.\times 1.2 \mu \mathrm{m}^{3}\right)$ supported by a long $(\sim 300 \mu \mathrm{m}$ in length), narrow $(20 \mu \mathrm{m}$ in width) beam on both ends, with a cavity underneath. Figure 1(a) sketches the device. AC current flows through the gold-plated suspended structure under the influence of an external magnetic field $(\sim 1 \mathrm{~T})$, driving the microplate to in-plane resonance at $140 \mathrm{kHz}$ via Lorentz law. This frequency will be used throughout this work.

The suspended structure was fabricated in three steps: metal layer deposition, photolithography, and etching. Deposition of a metallic layer $(0.18 \mu \mathrm{m}$ of $\mathrm{Au}$ on $20 \mathrm{~nm}$ of $\mathrm{Cr})$ was sputtered on a wafer coated with silicon nitride $(1 \mu \mathrm{m})$. Then, photoresist of $1 \mu \mathrm{m}$ was spincoated and UV patterned to define the dimension of the electrode (microplate and supporting beams at both ends), followed by development. To ensure a sharp contour at the microplate's edges, a metal layer was allowed to overhang the nitride substrate by $\sim 2 \mu \mathrm{m}$ [Fig. 1(b)]. Nonsharp edge was also fabricated with the gold layer ends at $\sim 2 \mu \mathrm{m}$ short of the plate's edge, exposing the silicon nitride structure underneath. The metal portion unprotected by photoresist was removed by chromium and gold etchant. To define the cavity $(\sim 750 \mu \mathrm{m}$ along the beam direction, by $500 \mu \mathrm{m}$ width) underneath the suspended structure, repeat of the photolithographic process was made. Finally, suspension of the plate/beam structure required removal of exposed silicon nitride using reactive ion etching, followed by potassium hydroxide to etch the unwanted silicon. The microdevice was tested in a small PMMA container with an unbounded fluid (water) space 

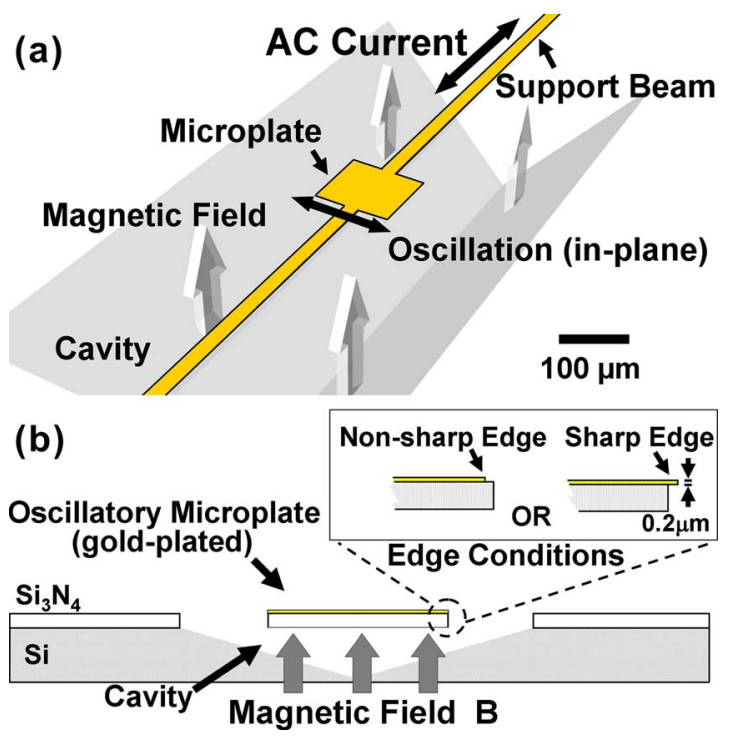

FIG. 1. (Color online) Sketch of the microdevice. (a) Suspended microplate $\left(100 \times 100 \times 1.2 \mu \mathrm{m}^{3}\right)$ in resonance $(140 \mathrm{kHz})$. Plate oscillation is due to Lorentz induced force from ac current flowing through the structure above an external magnetic field. (b) Edge-on view showing the sharp edges of the microplate. The sketch shows the $\mathrm{Si}_{3} \mathrm{~N}_{4}$ structure, and the cavity underneath. On the upper-right, the sketch shows the sharp edge and the nonsharp edge conditions of the microplate. The length of the metal layer overhang substrate is $\sim 2 \mu \mathrm{m}$ with thickness of $0.2 \mu \mathrm{m}$.

which the depth of the fluid $\sim 1500$ times than the $\delta_{\mathrm{ac}}$.

Figure 2(a) presents micrograph of the microvortices with diameter of $\sim 100 \mu \mathrm{m}$, as made evident through polystyrene tracer particles $(10 \mu \mathrm{m})$ in near-circular trajectory. The dominant effect of the oscillatory microplate with sharp edges is generation of a pair of two-dimensional (2D), small-
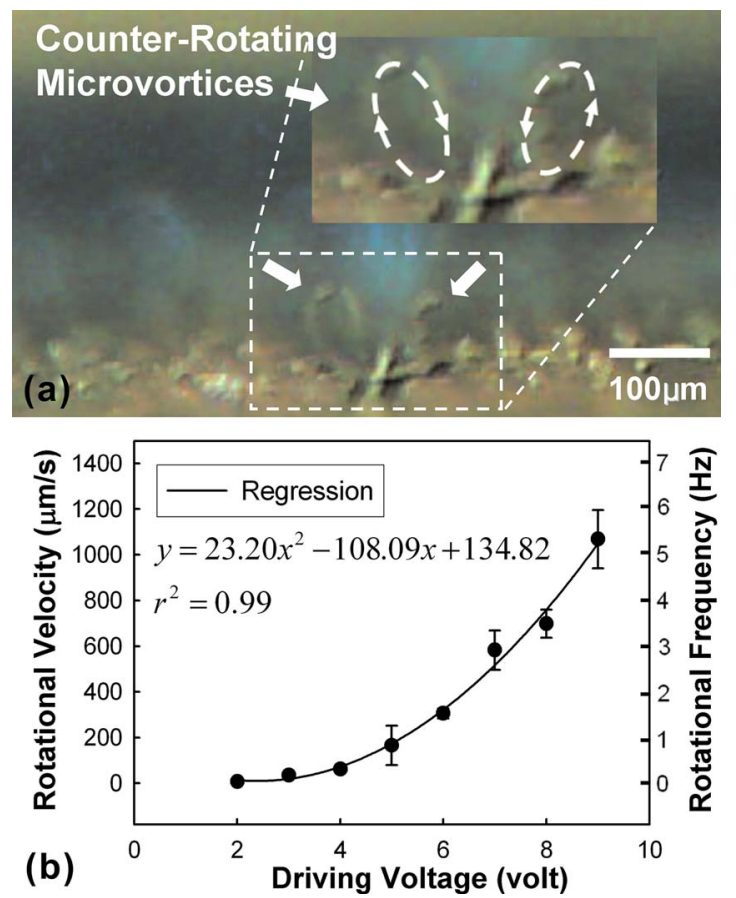

FIG. 2. (Color online) (a) Micrograph revealing counter-rotating microvortices (arrows shown) via tracer particles in water. Diameter of each vortex is of order of plate dimension $(100 \mu \mathrm{m})$. (b) Measured rotational velocity of a vortex vs excitation voltage (peak-to-peak). Frequency, or rev/s, is also shown on the right ordinate. Data suggest the microvortices are very controllable and robust.

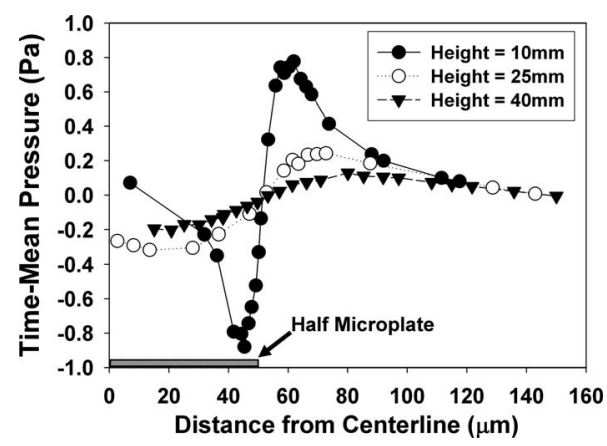

FIG. 3. Computational results of the microvortices. Time-mean pressure distributions are just above the plate at heights of 10,25 , and $40 \mu \mathrm{m}$.

scale, counter-rotating microvortices. Movie images show majority of the particles are initially drawn from far above the plate toward the plate center, then, as they approach the surface, are directed toward the two edges, ejected outward, looped upward, and recirculated back toward the plate center, repeating the trajectory. A small number of particles, however, are ejected far beyond the plates' edges and never to return. Figure 2(b) obtained from postprocessing of images in Fig. 2(a), presents the velocity of tracer particles while trapped by a vortex at different ac voltage applied across the microplate. Distinct trend of increase of vortex velocity (particles in circular motion) with voltage is evident. At maximum voltage of $9 \mathrm{~V}_{\mathrm{pp}}$ (peak-to-peak) tested, velocity of $1 \mathrm{~mm} / \mathrm{s}$, or $\sim 5 \mathrm{~Hz}(5 \mathrm{rev} / \mathrm{s})$, is attained. A threshold voltage of about $2 \mathrm{~V}_{\mathrm{pp}}$ is also evident, since this low driving voltage is probably insufficient to drive the microplate oscillation. Once generated, data show the microvortices are robust and controllable.

Complementary results from simulation (Comsol Multiphysics Inc.) support the experimental findings of vortices generation and provide further insight to the flow physics involved. To interrogate the numerics further, Fig. 3 plots the computed time-mean pressure (averaged from unsteady pressure) above the oscillatory plate under $1 \mu \mathrm{m}$ displacement at $140 \mathrm{kHz}$ in water $\left(\rho=1.00 \mathrm{~g} / \mathrm{cm}^{3}, \nu=0.0098 \mathrm{~cm}^{2} / \mathrm{s}\right)$. Results are plotted from the plate centerline outward towards the edge $(x=50 \mu \mathrm{m})$ and beyond at constant height of 10 , 25 , and $40 \mu \mathrm{m}$. Results indicate larger pressure variation at $10 \mu \mathrm{m}$ height than that of further away from the plate. At $10 \mu \mathrm{m}$ height, pressure decreases from plate center $(x=0)$ toward the plate's edge. Across the edge, however, strong adverse pressure gradient from 45 to $60 \mu \mathrm{m}$ is evident, suggesting the flow experiences strong deceleration along the $x$ direction. This corresponds to the flow begins to recirculate (flowing upward). At height of 25 and $40 \mu \mathrm{m}$, this adverse pressure is still evident but much less pronounced. The pressure approaches to ambient far from the plate (beyond $80 \mu \mathrm{m})$. Computed time-mean vorticity field (not presented) reveals distinct vorticity at $x=-50 \mu \mathrm{m}$ (left plate edge) and $50 \mu \mathrm{m}$ (right plate edge), with magnitude equal but opposite sign-indicative of the pair of counter-rotating microvortices.

To probe the importance of the plate's edge further, effect of edge sharpness on flow structure was studied via an identical microdevice except with a nonsharp edge on one side of the plate and a sharp edge on the other. Results in Fig. 4(a) show a 2D steady microvortex is found only on the side of the plate with sharp edge. This result confirms that the 

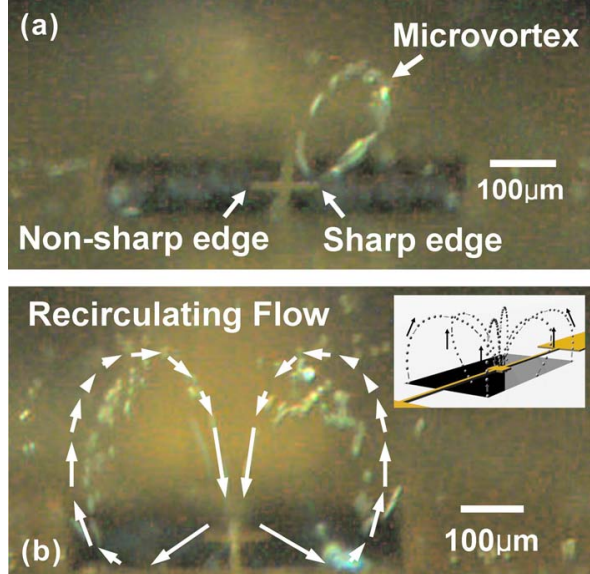

FIG. 4. (Color online) (a) Micrograph showing the flow with a microvortex on the right generated by a sharp edge. Movie images show 3D recirculating flow by a nonsharp edge on the left. (The 3D circulating flow is not as clear because tracer particles are drawn towards the $2 \mathrm{D}$ vortex on the right.) (b) Flow structure with nonsharp plate edges. Micrograph shows the tracer particles approach the plate center, then ejected outward and into the cavity prior to recirculating back towards the plate again. The inset sketches the 3D flow field. Interaction with local geometry (cavity) results in the much large (order of cavity) flow structure.

plate's edge plays a crucial role in formation of streaming flow.

The fundamental flow structure with nonsharp plate's edge is different than that with a sharp edge. For nonsharp edges, Fig. 4(b) suggests the flow structure exhibits 3D, deterministic, and large scale (order of cavity dimension). Tracer particles are drawn toward the plate center, rapidly ejected outward and into the cavity, then recirculate toward the plate again. This pattern is vastly different than that of Fig. 2(a). Movie images of Fig. 4(a) also show 3D, largescale, recirculating flow is present for nonshape edge, but not as evident as in Fig. 4(b). The reason is that tracer particles in Fig. 4(a) are easier to be drawn by the 2D microvortex (on the right) than toward the 3D circulating flow (on the left).

Results reveal the importance of the all-critical microplate's edge. Sharp edges ensure 2D microvortices, and a nonsharp edge generates no vortex but 3D recirculating flow. The dependence of sharp and nonsharp edge conditions can be explained by the dimensionless parameter $\varepsilon(=s / t)$, where $s$ is the displacement amplitude and $t$ the thickness at the plate's edge. Under the fixed oscillating frequency $(140 \mathrm{kHz})$ and driving voltage, $s$ is fixed $\left(\sim 0.5 \mu \mathrm{m}\right.$ at $\left.5 \mathrm{~V}_{\mathrm{pp}}\right)$ for both edge conditions. Parameter $t$ for the sharp edge is characterized by the thickness of the overhanging gold layer $(0.2 \mu \mathrm{m})$; for the nonsharp edge is the thickness of the microplate $(1.2 \mu \mathrm{m})$. Hence, $\varepsilon \geqslant 1$ for the sharp edge, forming 2D microvortices; $\varepsilon<1$ for non sharp edge, resulting in 3D recirculating flow.

The microdevice presented provides a mechanical strategy for generating circulating flow pattern in a microfluidic system. The merits of the microvortices includes (1) tunable flow structure by designing a specific edge condition of the microplate-either $\varepsilon \geqslant 1$ for 2D microvortices or $\varepsilon<1$ for $3 \mathrm{D}$ recirculating flow, (2) operation of the device is believed to be essentially independent of properties of fluid or embedded bioparticles, (3) low operating voltage, and (4) robust and controllable. These features should make the microdevice attractive for a wide range of applications in microfluidics.

The authors are grateful for support from the National Science Council under grants from the National Project on Nanoscience and Technology (NSC 94-2120-M-002-014, NSC 95-2120-M-002-006, and NSC 96-2120-M-002-002).

${ }^{1}$ H. A. Stone, A. D. Stroock, and A. Ajdari, Annu. Rev. Fluid Mech. 36, 381 (2004).

${ }^{2}$ G. M. Whitesides, Nature (London) 442, 368 (2006).

${ }^{3}$ P. S. Dittrich, K. Tachikawa, and A. Manz, Anal. Chem. 78, 3887 (2006).

${ }^{4}$ A. D. Stroock, S. K. W. Dertinger, A. Ajdari, I. Mezic, H. A. Stone, and G. M. Whitesides, Science 295, 647 (2002).

${ }^{5}$ F. Bottausci, C. Cardonne, C. Meinhart, and I. Mezic, Lab Chip 7, 396 (2007).

${ }^{6}$ P. Takhistov, K. Duginova, and H. C. Chang, J. Colloid Interface Sci. 263, 133 (2003).

${ }^{7}$ N. G. Green, Anal. Bioanal. Chem. 382, 891 (2005).

${ }^{8}$ Y.-K. Lee, L. M. Lee, W. L. W. Hau, and Y. Zohar, J. Microelectromech. Syst. 16, 58 (2007).

${ }^{9}$ P. Tierno, T. H. Johansen, and T. M. Fischer, J. Phys. Chem. B 111, 3077 (2007).

${ }^{10}$ M. Kostur, M. Schindler, P. Talkner, and P. Hanggi, Phys. Rev. Lett. 96 014502 (2006).

${ }^{11}$ S. N. Wang, X. Hu, and L. J. Lee, J. Am. Chem. Soc. 129, 254 (2007).

${ }^{12}$ J. Holtsmark, I. Johnsen, T. Sikkeland, and S. Skavlem, J. Acoust. Soc. Am. 26, 26 (1954).

${ }^{13}$ W. P. Raney, J. C. Corelli, and P. J. Westervelt, J. Acoust. Soc. Am. 26 1006 (1954).

${ }^{14}$ W. L. M. Nyborg, Physical Acoustics: Principles and Methods (Academic, New York, 1964)

${ }^{15}$ H. Schlichting, Boundary-Layer Theory (McGraw-Hill, New York, 1979).

${ }^{16}$ N. T. Nguyen and R. M. White, Sens. Actuators, A 77, 229 (1999).

${ }^{17}$ R. H. Liu, J. N. Yang, M. Z. Pindera, M. Athavale, and P. Grodzinski, Lab Chip 2, 151 (2002) 